\title{
Abhava pratinidhi dravya: A Comparative Phytochemical Study of Bharangi and Kantakari
}

\author{
Research Article
}

\section{Choudhari D D ${ }^{1 *}$, Kulkarni Y R ${ }^{2}$, Visave $\mathbf{P ~ B}^{3}$}

\author{
1. Ph.D Scholar, 2. Ph.D. Guide and Professor, Department of Dravyaguna, \\ P.D.E.A'S College of Ayurved and Research Centre, Nigdi, Pune. \\ 3. Associate Professor, Siddhakala Ayurved college, Sangamner, Nagar.
}

\begin{abstract}
Abhava pratinidhi dravya excites the scientific curiosity concerning the Ayurvedic principles behind selection of the substitute drug. Present study had reported a comparative phytochemical study of one such Ayurvedic substitution pair: Solanum surattense Burm.f., a substitute for Clerodendrum serratum L. The study was conducted to understand the logic behind the Abhava pratinidhi dravya (Drug substitution). The strategy was to scrutinize and compare their Ayurvedic properties by literary studies and test the phytochemical profile of these two herbs for biochemical similiarties and differences. On analyzing their Ayurvedic pharmacology (Dravyaguna properties), findings show that both the drugs possess katu and tikta rasa, but Bharangi is katu rasa dominant and Kantakari is tikta rasa dominant drug. Along with this, both drug possess laghu, ruksha and ushna guna in common. Kantakari which contain different properties i.e. sara and tikshna guna. Both drug possess katu vipaka and ushna veerya. Both drugs had been stated to treat majority of respiratory ailments. Phytochemical observations suggest that in Bharangi root extract alkaloids and tannins was present whereas Kantakari root extract was rich in flavonoids content. Saponins was present in maximum amount in both the plant extracts. Despite taxonomically unrelated and morphologically dissimiliar, Bharangi has been substituted by Kantakari drug. In Ayurveda, more importance was given to pharmacological properties of raw drug rather than its botanical classification. Further comparative preclinical studies and bio-equivalence clinical studies has been needed to explore the different pharmacological properties.
\end{abstract}

Key Words: Abhava pratinidhi dravya, Ayurved materia medica, Bharangi, Clerodendrum serratum( Linn) Moon, Drug substitution, Kantakari, Solanum surattense Burm. f., Substitute.

\section{Introduction}

Classical Ayurveda had recommended use of a functionally similar substitute. Abhava pratinidhi dravya is one of the concept mentioned in Ayurvedic texts like Yogratnakar, Bhavaprakasha and Bheshajya Ratnavali.(1,2,3) Bhavaprakasha was the first who mentioned this concept in 16 th century A.D. and then repeated subsequently in forthcoming Ayurvedic literature. Abhava pratinidhi dravya (drug substitution) concept is based upon the dravyaguna vigyana principles. For substitution, each drug is identified and characterized on the basis of its rasapanchaka attributes i.e. Rasa (taste), Guna (properties), Veerya (potency), Vipaka ( metabolism), Prabhava (specific action) and Karma (pharmacological action).(2,3) In the present article, a preliminary study of one such Ayurvedic substitution

\section{* Corresponding Author:}

\section{Choudhari D D}

Associate Professor,

Department of Dravyaguna Vigyana

S.S.T Ayurved College, Sangamner

Dist. Ahmednagar, Maharashtra,412044, India

Email Id: aditi343@gmail.com pair: Kantakari (Solanum surattense Burm. f., Solanaceae), a common drug for the seasonally available species, Bharangi (Clerodendrum serratum L., Verbenaceae).

The objective this study was to analyze and compare Ayurvedic properties by literary studies and test the phytochemical profile of these two herbs for biochemical similiarities and differences. No previous studies had been recorded to compare these two drugs chemical profiles. Kantakari and Bharangi, despite being taxonomically unrelated, are not only similar in Ayurvedic pharmacology (Dravyaguna) profile but also phytochemically.

\section{Methodology}

After brainstorming with the Supervisor, a structured strategy was planned. APD (Abhava pratinidhi dravya) was enlisted from the Ayurvedic texts, Bhavaprakasha Nighantu, (2) Yogratnakar (1) and Bhaishajya Ratnavali (3). Kantakari and Bharangi was selected from the list due to striking dissimilarities in taxonomy. The comparison was done at two different levels i.e. Dravyaguna (Ayurvedic pharmacology), chemical profiles (using phytochemical screening). 
Choudhari D D et.al., Abhava pratinidhi dravya: A Comparative Phytochemical Study of Bharangi and Kantakari

\section{Plant material}

Field samples of Solanum surattense Burm.f. and Bharangi Clerodendrum serratum L. were collected and authenticated by an authorized field botanist and an Ayurvedic practitioner. Voucher specimens were deposited with the Herbarium at B.S.I. Pune. The herbarium voucher specimen number of Solanum surattense Burm.f., was DDC 02 and of Clerodendrum serratum L. was DDC 01 respectively. (Ref. letter no. BSI/WRC/IDEN.CER./ 2020/H3 Dated 28/02/2020.)

Figure 1: Clerodendrum serratum (L.) Moon

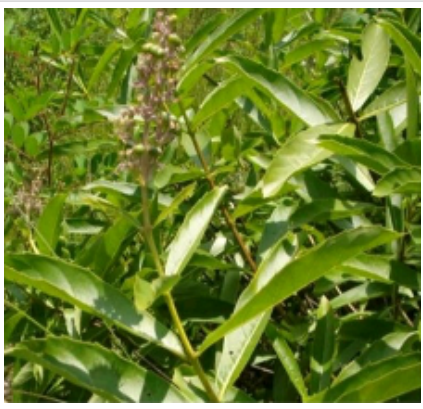

Plant

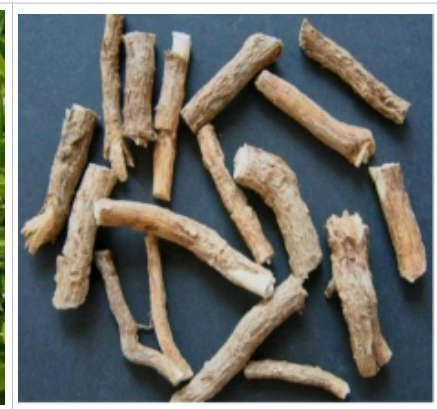

Root
Figure 2 : Solanum surattense Burm. f.

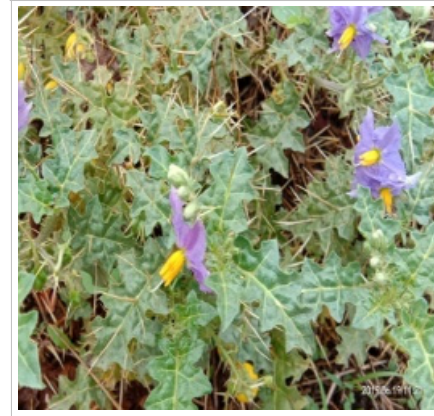

Plant

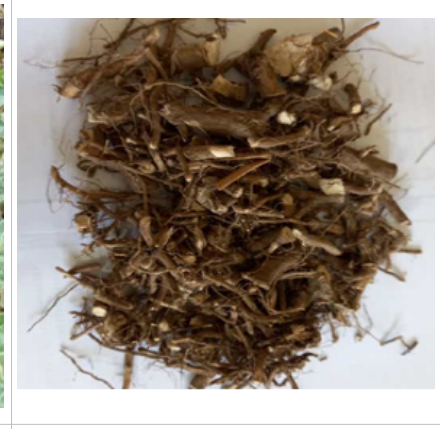

Root

\section{Pharmacognostical Studies}

Clerodendrum serratum (Linn.) Moon (Fam. Verbenaceae); is accepted by Ayurvedic
Pharmacopeia of India as Bharangi. It is blue flowered shrub,which is widely distributed throughout India. (4) It is 0.9-2.4 m. high, glabrous,bluntly quadrangular stem, opposite and ternate leaves, mostly serrate, stout and $6 \mathrm{~mm}$. long. Flowers are numerous, in lax pubescent dichotomous cymes. (5)

Kantakari also recognized as plant of Solanum surattense Burm. f., (Fam. Solanaceae, perennial, by Ayurvedic Pharmacopeia of India, very prickly diffused herb of waste land, available throughout India.(6) Stem zigzag, with numerous branches, younger ones clothed with dense stellate tomentum; compressed prickles,, straight, yellow, glabrous and shining, often exceeding $1.3 \mathrm{~cm}$ long. Leaves $5-10$ by $2.5-5.7 \mathrm{~cm}$., ovate or elliptic, sinuate or subpinnatifid stellately hairy beneath. Flowers in extra-axillary, few flowered cymes sometimes reduced to a single flower. Berry yellow, 1.3-2 $\mathrm{cm}$ in diameter with green veins, surrounded by enlarged calyx. Seeds glabrous and $2.5 \mathrm{~mm}$ in diameter. (7)

\section{Dravyaguna studies}

Raspanchaka i.e. Ayurvedic pharmacological parameters of Bharangi and Kantakari was compiled from Charaka Samhita $(8,9)$, Sushruta Samhita $(10,11)$, Ashtanga Hridaya (12,13), Yogaratnakar(1) and Bhaishajya Ratnavali(3) and from Lexicons like Bhavaprakasha, (14,15) Dhanvantari $(16,17)$, Raja $(18,19)$ and Kaiyadeva Nighantu. (20,21). Contemporary scholars understanding was also consider like work of authors Deshpande et. al $(22,23)$ and Sharma $(24,25)$ and Shastry respectively. $(26,27)$

Information about similiarities and differences based on Ayurvedic Pharmacology (Dravyaguna) was analysed in terms of their Rasa( Taste), Guna ( Properties), Veerya( Potency) and Vipaka ( Metabolic stage). Two extensive review article has already been published on this topic. $(28,29)$.

Table 1: Summary of properties and actions (Raspanchakas) of Bharangi and Kantakari

\begin{tabular}{|c|c|c|c|c|}
\hline Sr. No. & Properties & Bharangi & Kantakari & Similarities \\
\hline 1 & $\begin{array}{l}\text { Rasa } \\
\text { (Taste) }\end{array}$ & $\begin{array}{l}\text { Katu }(14,26) \text { (Pungent), Tikta(14,26) } \\
\text { (Bitter) }\end{array}$ & $\begin{array}{l}\text { Tikta }(15,27) \text { (Bitter), Katu(15,27) } \\
\text { (Pungent) }\end{array}$ & $\begin{array}{l}\text { Tikta (Bitter), } \\
\text { Katu (Pungent) }\end{array}$ \\
\hline 2 & $\begin{array}{l}\text { Guna } \\
\text { (Property) }\end{array}$ & $\begin{array}{l}\text { Laghu(14,26) (Light), } \\
\text { Ruksha(14,26) (Dry), } \\
\text { Ushna }(14,26) \text { (Hot) }\end{array}$ & $\begin{array}{l}\text { Laghu(15,27) (Light), } \\
\text { Ruksha(15,27) (Dry), } \\
\text { Ushna (15) (Hot), } \\
\text { Sara (15) (Increases intestinal } \\
\text { activity), } \\
\text { Tikshna (27) (Irritable) }\end{array}$ & $\begin{array}{l}\text { Laghu (Light), } \\
\text { Ruksha (Dry), } \\
\text { Ushna (Hot) }\end{array}$ \\
\hline 3 & $\begin{array}{l}\text { Virya } \\
\text { (Potency) }\end{array}$ & Ushna(14,26) (Hot) & Ushna $(15,27)(\mathrm{Hot})$ & Ushna (Hot) \\
\hline 4 & $\begin{array}{l}\text { Vipaka } \\
\text { (Stage of } \\
\text { digestion) }\end{array}$ & Katu $(14,26)$ (Pungent) & Katu $(15,27)$ (Pungent) & Katu (Pungent) \\
\hline
\end{tabular}


5 Karma $\quad$ Kapha-marutaghna(14,26) (Reduces $\quad$ Pachani(15) (Digests undigested

(Actions) Kapha-vata), Pachani(14) (Digests undigested food material),

Dipana(14) (Increases digestive fire), Gulmanashaka(14) (Decreases lump), Raktadoshanashaka (14) (Decreases blood disorder), Shothahara (14) (Decreases inflammation ), Kasahara(14,26) (Anti-tussive ), Kaphashwasahara(14) (Anti-asthmatic), Jwarahara(14,26) (Anti-pyretic ), Pinasa nashaka(14) (Lowers upper respiratory tract infection ), Marutahara(14) (Decreases vata ).

6 Indications Shwasa(14,26) (Asthma),

Kasa $(14,26)$ (Cough ), Pratishyaya(14) (Upper respiratory infection), Yakshmaja Kasa(14) (), Vata-kaphaja Jwara(14,26) (Fever), Amavata(14) (Rheumatoid arthritis ), Shotha (26) (inflammation), Vrana (26) (Wound), Krumi (26) (Antibacterial), Daha (26) (Heat reducing), Pinasa nashaka(26) (Lowers upper respiratory tract infection ).

food material), Dipana(15)

(Increases digestive fire),

Kasahara(15) (Anti-tussive ),

Kapha-shwasahara(15) (Anti-

asthmatic ), Jwarahara(15) (Anti-

pyretic ), Anilnashaka (15)

( Stabilizes vata), Pinasa

nashaka(15) (Lowers upper

respiratory tract infection ),

Parshwapida (15) ( pain in upper

abdomen), Kruminashaka (15)

(Anti-bacterial),

Hridyaroganashaka(15) ( Heart

diseases)

Kasa(15,27) (Cough ),

Shwasa(15,27) (Asthma ),

Pratishyaya(15,27) (Upper

respiratory infection ), Jwara(15)

(Fever), Angamarda(15)

(Bodyache ), Parshwapida (15)

(Pain in upper abdomen ),

Hridyaroga(15) (Heart diseases ),

Admana (15) ( Bloating), Vibandha

(15) (Constipation ), Ashmari

$(15,27)$ (Renal

calculus ),Vamananashaka (15)

(Anti-emetic), Shotha (27)

(Inflammation), Hikka (27)

( Hiccough), Mutrakruchha (27)

( Difficulty in urination).

7 Part used Roots(26)
Roots, Seeds, Fruits, Panchanga,

Whole plant (27)
Dipana (Increases

digestive fire), Pachani

(Digests undigested

food material),

Kasahara (Anti-

tussive ), Kapha-

shwasahara (Anti-

asthmatic ), Jwarahara

(Anti-pyretic ), Pinasa nashaka (Lowers upper respiratory tract infection ).

Kasa (Cough ), Shwasa (Asthma ), Pratishyaya (Upper respiratory infection ), Jwara (Fever), Shotha (Inflammation).

Roots

Table 2: Physico-chemical Standards of Clerodendrum serratum and Solanum surattense

\begin{tabular}{|c|l|c|c|c|}
\hline Sr. No. & \multicolumn{1}{|c|}{ Tests } & Clerodendrum serratum & Solanum surattense & Comment \\
\hline 1 & Foreign Matter & Nil & Nil & Nil \\
\hline 2 & Moisture Content & $11.70 \%$ & $7.88 \%$ & Values as per API standard \\
\hline 3 & Ash Value & $3.97 \%$ & $5.52 \%$ & Values as per API standard \\
\hline 4 & Acid insoluble Ash & $0.93 \%$ & $1.94 \%$ & Values as per API standard \\
\hline 5 & Water soluble extractive value & $12.62 \%$ & $41.91 \%$ & More in Kantakari \\
\hline 6 & Alcohol soluble extractive value & $8.99 \%$ & $0.55 \%$ & More in Bharangi \\
\hline 7 & Ph Value (5\% solution) & 6.03 & 6.04 & Same \\
\hline 8 & Specific gravity & 1.00 & 1.00 & Same \\
\hline
\end{tabular}

Table 3: Qualitative chemical screening of Clerodendrum serratum and Solanum surattense

\begin{tabular}{|c|l|c|c|}
\hline Sr. No. & \multicolumn{1}{|c|}{ Phytoconstituents } & Clerodendrum serratum & Solanum surattense \\
\hline 1 & Alkaloids (Water) & + & - \\
\hline 2 & Cardiac Glycosides & - & - \\
\hline 3 & Anthraquinone & - & - \\
\hline & Glycosides & + & + \\
\hline 5 & Saponins & - & + \\
\hline 6 & Flavonoids & + & - \\
\hline
\end{tabular}

\section{Discussion}

Abhava and Abhava Pratinidhi Dravya from 16 th to 19 th century of Ayurveda referred texts has not mentioned any logic of selection of substitutes, parts used and details of usage in what pharmaceutical form. Two drugs can be substituted for each other only on the basis of botanical, pharmacognostical, phytochemical and pharmacological similiarities or by Linking and observing, accessing its chemistry and bioactivity.
An analysis of the Table 1indicates that the Dravyaguna qualities of the two drugs i.e. Rasa (Taste), Guna (Properties), Vipaka (Stage of digestion), Veerya (potency) and Karma (Actions) were very similar. On analyzing their Ayurvedic pharmacology (Dravyaguna properties), findings show that both the drugs possess katu and tikta rasa, but Bharangi is katu rasa dominant and Kantakari is tikta rasa dominant drug. Along with this, both drug possess laghu, ruksha and ushna guna in common. Kantakari which contain different properties i.e. sara and tikshna guna. Both drugs possess katu 
Choudhari D D et.al., Abhava pratinidhi dravya: A Comparative Phytochemical Study of Bharangi and Kantakari

vipaka and ushna veerya. Both drugs have been stated to treat majority of respiratory ailments.

Table 2. shows the physico-chemical standard of the two drugs. Absence of foreign matter in both the drugs suggests purity of sample. All the physicochemical parameters tested were as per Ayurvedic Pharmacoepoeial Standard. $(4,6)$ On analyzing in detail, it was found that water soluble extractive value of Solanum surattense Burm. f. is higher $(41.91 \%)$ as compared to water soluble extractive value of Clerodendrum serratum (L.) Moon (12.62\%). In case of alcohol soluble extractive value, Clerodendrum serratum (L.) Moon (8.99\%) value is higher than alcohol soluble extractive value of Solanum surattense Burm. f. ( $0.55 \%)$. Ph value of both the drug is same i.e. 6.04. Both the drug have same Specific gravity i.e. 1.

Table 3. Phytochemical observations suggest that in Bharangi root extract alkaloids and tannins was present whereas Kantakari root extract was rich in flavonoids content. Saponins was present in maximum amount in both the plant extracts. Despite taxonomically unrelated and morphologically dissimiliar, Bharangi has been substituted by Kantakari drug. In Ayurveda, more importance was given to pharmacological properties of raw drug rather than its botanical classification. Further comparative preclinical studies and bio-equivalence clinical studies has been needed to explore the different pharmacological properties.

\section{Conclusion}

After analyzing and comparing Ayurvedic properties by literary studies it was found that both the drugs possess similar Ayurvedic pharmacological properties and could be substituted for each other. But the chemical profile of two drugs was completely different. Kantakari and Bharangi, also taxonomically unrelated but have similiar ayurvedic pharmacology (Dravyaguna) profile. From the above study it can be concluded that more integrative ayurvedic studies are needed to evaluate any herbal drug. Botanical classification is relevant for identification and quality control, but techniques to evaluate medicinal uses are more relevant for treatment/ practice purpose. Even though the current study is preliminary, it provides a different perspective that can be applied to study drug substitutes. For further scope of drug evaluation more comparative pre-clinical and clinical studies should be planned.

\section{References}

1. Shastry L. editor. Yogaratnakara. with Vidyotini Hindi commentary. 7 th ed. Varanasi; Chaukhamba Sanskrit Sansthan; 2002. 171p

2. Bhavprakash. Bhavprakasha with Vidyotini Hindi Commentary. Ed. Mishra Brahma Shankar. Vaishya Rupalalji. 7 th ed. Chaukhamba Sanskrit Sansthan; Varanasi; $1990.87 \mathrm{p}$

3. Mishra S.N. editor. Bhaishajyaratnavali of Kaviraj Govind Das Sen.1st ed.Varanasi; Chaukhambha Surbharti Prakashan; 2007. 80p
4. Anonymous. The Ayurved Pharmacopoeia of India. Part I. Vol.III. Ist ed. Govt. of India. Dept. of I.S.M.\& H.; 2001. 25-26p

5. Kirtikar K.R., Basu B.D. Indian Medicinal Plants. Vol.III. 2 nd ed. International Book Distributers Dehradun; 2005. 1948p

6. Anonymous. The Ayurved Pharmacopoeia of India. Part I. Vol.III. Ist ed. Govt. of India. Dept. of I.S.M.\& H.; 2001.77-80p

7. Kirtikar K.R., Basu B.D. Indian Medicinal Plants. Vol.III. 2 nd ed. International Book Distributers Dehradun; 2005. 1759p

8. Acharya Charak. Charak samhita. Ayurved Dipika Commentary. Vol.II. ed. Pandit Kashinath Shastri. Gangasahaya Pandeya. Chaukhambha Sanskrit Sansthana; Varanasi; 2011. 476p

9. Acharya Charak. Charak samhita. Ayurved Dipika Commentary. Vol.II. ed. Pandit Kashinath Shastri. Gangasahaya Pandeya. Chaukhambha Sanskrit Sansthana; Varanasi; 2011. 458p

10. Acharya Sushruta. Sushruta Samhita. Vol.I. ed. Shrikantha Murthy K.R. (Translator). Chaukhambha Orientalia; Varanasi; 2012. 302p

11. Acharya Sushruta. Sushruta Samhita. Vol.II. ed. Shrikantha Murthy K.R. (Translator). Chaukhambha Orientalia; Varanasi; 2012. 87p

12. Acharya Vagbhat. Ashtang Hridaya. Vol.I. Vidyotini Hindi Commentary. Kaviraj Atridev Gupta. Chaukhambha Orientalia; Varanasi; 2012. 415p

13. Acharya Vagbhat. Ashtang Hridaya. Vol.I. Vidyotini Hindi Commentary. Kaviraj Atridev Gupta. Chaukhambha Orientalia; Varanasi; 2012. 416p

14. Chunekar K.C. Bhavaprakasha Nighantu of Bhavamisra. 2 nd edition. Varanasi; Chaukhambha Bharati Academy; 2010. 98p

15. Chunekar K.C. Bhavaprakasha Nighantu of Bhavamisra. 2 nd edition. Varanasi; Chaukhambha Bharati Academy; 2010. 276p

16. Dwivedi B.K. Dhanwantari Nighantu. Guduchyadi Varga. Chaukhambha Krishnadas Academy; Varanasi; 2008. 102p

17. Dwivedi B.K. Dhanwantari Nighantu. Guduchyadi Varga. Chaukhambha Krishnadas Academy; Varanasi; $2008.108 \mathrm{p}$

18. Narhari. Raj Nighantu. Edited by Tripathi Indradev. 5 th ed. Guduchyadi Varga. Chaukhambha Krishnadas Academy; Varanasi; 2006. 03p

19. Narhari. Raj Nighantu. Edited by Tripathi Indradev. 5 th ed. Shatavhyadi Varga. Chaukhambha Krishnadas Academy; Varanasi; 2006. 30p

20. Kaiyadev. Kaiyadev Nighantu. Priyavata Sharma ed. Oushadhi Varga. Chaukhambha Orientalia; Varanasi; 2009. 210p

21. Kaiyadev. Kaiyadev Nighantu. Priyavata Sharma ed. Oushadhi Varga. Chaukhambha Orientalia; Varanasi; 2009. 52p. www.niimh.nic.in

22. Deshpande. A.P. Javalgekar R.R. Ranade Subhash. Dravyagunavidnyan (Part I\&II). Proficient Publishing House; Pune; 2009. 716p

23. Deshpande. A.P. Javalgekar R.R. Ranade Subhash. Dravyagunavidnyan (Part I\&II). Proficient Publishing House; Pune; 2009. 697p 
24. Priyavrat Sharma. Dravyaguna Vigyana. Vol. II. Chaukhambha Bharati Academy; Varanasi; 2005. $298 \mathrm{p}$

25. Priyavrat Sharma. Dravyaguna Vigyana. Vol. II. Chaukhambha Bharati Academy; Varanasi; 2005. $280 \mathrm{p}$

26. Sastry J.L.N. Nesari T.M. A Textbook of Dravyaguna Vijnana. Varanasi; Chaukhambha Orientalia; 2018. 54p

27. Sastry J.L.N. Nesari T.M. A Textbook of Dravyaguna Vijnana. Varanasi; Chaukhambha Orientalia; 2018. 147p
28. Choudhari Deepali.D., Y.R.Kulkarni. Experimental Pharmacology of Clerodendrum serratum (Linn.) Moon- A Review Update. International Journal of Applied Ayurved and Research 2019; 4(3); 265-270.

29. Deepali D. Choudhari, Y.R.Kulkarni. A Critical review on Bharangi [Clerodendrum serratum (Linn.) Moon] with special reference to Ayurvedic Nighantus (Lexicons). National Journal of Research in Ayurved Science 2019; 7(7);1-13. 